\section{Broadband CARS Microscopy}

\section{Marcus T. Cicerone and Tak W. Kee}

Polymers Division, National Institute of Standards and Technology, Gaithersburg, MD

cicerone@nist.gov

A major challenge in optical microscopy is to develop techniques with high spatial resolution, sensitivity, and chemical specificity. The latter, chemical specificity, is typically achieved through some form of labeling, which has potential to alter the nature of the sample under investigation. Raman or infrared (IR) microscopy can be utilized to image samples in their natural form using molecular vibrations as a contrast mechanism. IR microscopy suffers from spatial resolution issues, and spontaneous Raman microscopy suffers from low scattering cross-sections, so that high laser power is often required, introducing the possibility of sample photo-damage. Scattering cross-sections for Coherent Anti-Stokes Raman Scattering (CARS) are typically several orders of magnitude greater than those of spontaneous Raman Scattering. This, in addition to the high spatial resolution inherent in nonlinear optical microscopy, has led CARS microscopy to begin emerging as a powerful, noninvasive technique for biological and material imaging.

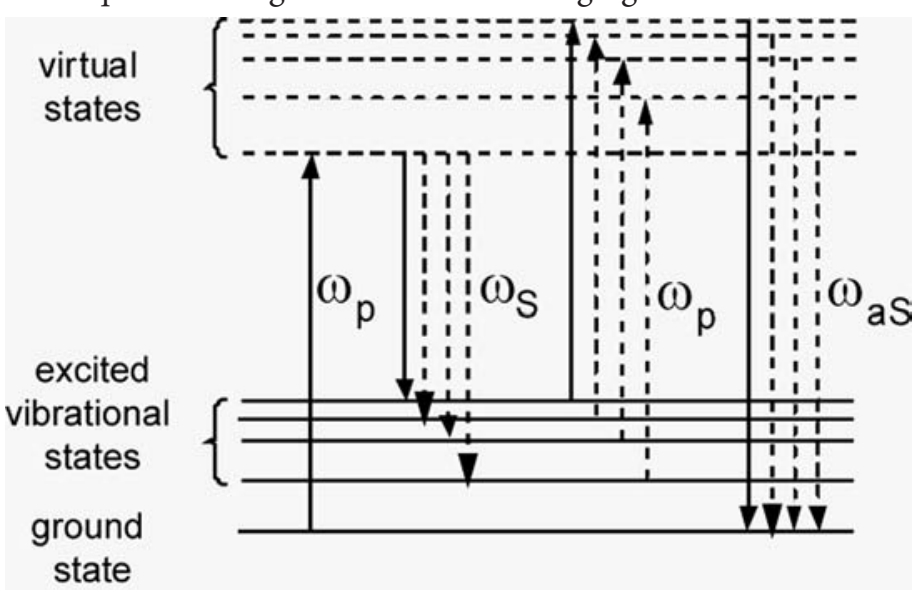

Figure 1. Energy level diagram for single frequency CARS process (solid vertical arrows) and Broadband CARS (solid and dashed vertical arrows).

CARS microscopy utilizes pulsed laser light of two frequencies to detect molecular vibrations. Pump light, with a frequency of $\omega_{\mathrm{p}}$, is mixed with Stokes light at $\omega_{\mathrm{S}}$ in the sample. When the frequency difference $\left(\omega_{\mathrm{p}}-\omega_{\mathrm{s}}\right)$ is resonant with a vibrational state in the sample, that state gets coherently populated. A portion of the pump light scatters off the coherent vibrations within the sample to produce the anti-Stokes light at $2 \omega_{\mathrm{p}}-\omega_{\mathrm{s}}$. Figure 1 is an energy level diagram that describes the CARS process.

The initial work on CARS microscopy dates back to 1982, when it was used to resolve detailed structure of onion-skin cells. ${ }^{1}$ In 1999, CARS microscopy was used in a collinear geometry and since then significant progress has been made, including imaging of live cells and photoresist materials. ${ }^{2}$ Today, there are two general directions in which CARS microscopy is being taken actively. One involves generation and detection of signal in a narrow spectral band to achieve noninvasive video rate imaging, usually of biological materials. The other direction is towards hyperspectral imaging. Generation and detection of signal in a broader spectral range provides for high chemical resolving power, but at a slower rate of image acquisition. In the broadband work up to now, a bandwidth of $\approx 200 \mathrm{~cm}^{-1}$ has been achieved and CARS microscopy has been used to image and distinguish two distinct chemical species simultaneously, providing a limited degree of chemical specificity. An increased breadth of spectral sensitivity would be needed to endow CARS microscopy with the ability to distinguish a large number of chemical species. Particularly, a broadband CARS technique that spectrally covers the Raman fingerprint region $\left(800 \mathrm{~cm}^{-1}\right.$ to 1800 $\mathrm{cm}^{-1}$ ) or beyond will allow for chemical identification of multiple species, and provide for the possibility of tracking subtle changes in complex systems, such as biological cells.

Figure 2a is a CARS spectrum of benzonitrile obtained with a broadband CARS microscope, the details of which we have recently reported. ${ }^{3}$ With this instrument, we can acquire spectra in the range of $500 \mathrm{~cm}^{-1}$ to $3100 \mathrm{~cm}^{-1}$ with good spatial resolution and reasonably fast pixelation rates (17 ms signal acquisition). The broadband CARS spectra are generated by using broadband Stokes light, as indicated by the dashed vertical arrows in Figure 1. The instrument utilizes a single $150-\mathrm{fs}$ unamplified Ti:sapphire laser. The laser output was divided into two parts; one was used as pump $\left(\omega_{\mathrm{p}}\right)$, and another part was focused into a tapered silica fiber to generate broadband Stokes light $\left(\omega_{S}\right)$. Use of a single laser practically eliminates temporal jitter between pump and Stokes light. The pump and Stokes light, having powers of $13 \mathrm{~mW}$ and $10 \mathrm{~mW}$ respectively, were combined in a dichroic beamsplitter and directed into a 0.8 NA microscope objective. The sample was scanned with a motorized $\mathrm{x}-\mathrm{y}$ stage and the broadband CARS signal was collected with a $0.5 \mathrm{NA}$ objective. A CCD camera mounted on a spectrograph was used for signal detection after spectral filtering.

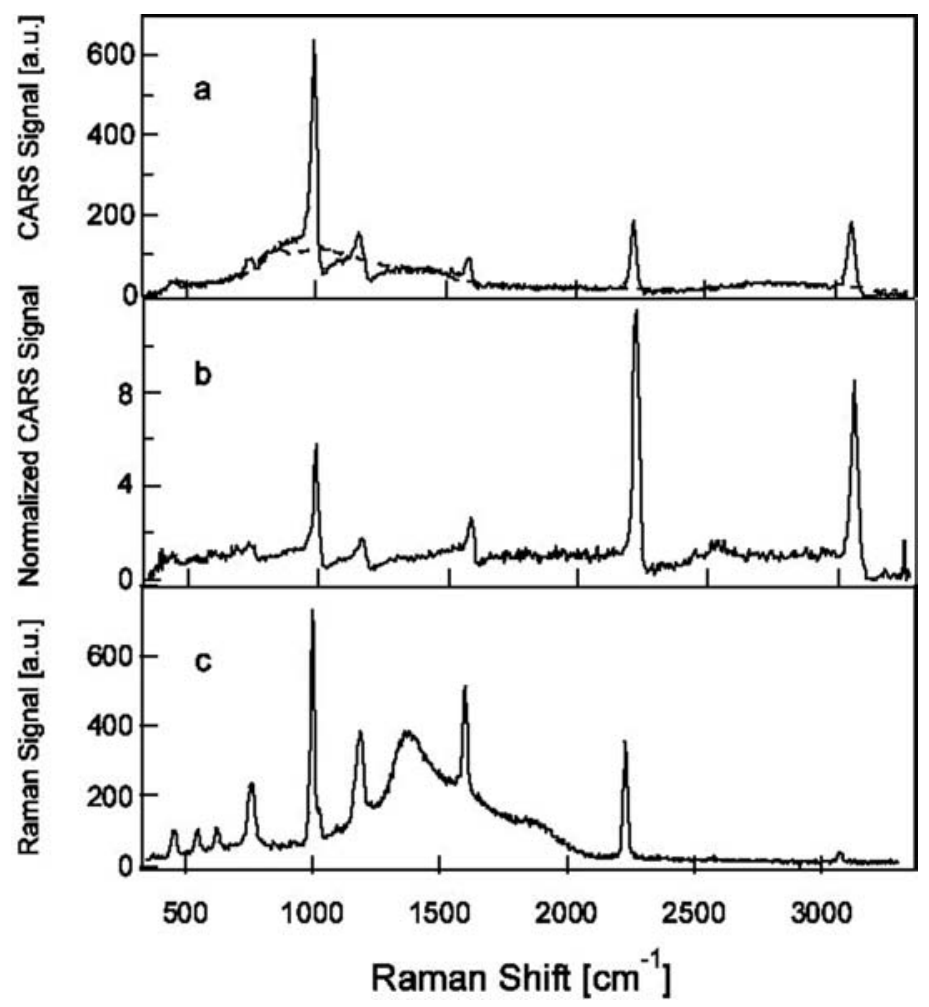

Figure 2. Broadband CARS spectrum of benzonitrile. Panel a: Raw CARS spectrum (solid line), and nonresonant background (dashed line). Panel b: Ratio of CARS spectrum to nonresonant background (solid line). Panel c: Spontaneous Raman spectrum obtained under identical laser flux (23 $\mathrm{mW}$, but all in pump light). 
With the release of Soft Imaging System's new TEM side-mounted camera MORADA, it is time to say good-bye to an old and trusty friend who has been with us since the beginning of electron microscopy: the photo plate.

Stop wasting time working in the darkroom, start saving time by electronic reporting within seconds of acquisition. The environment will thank you, too. And you can do that while maintaining or even improving on the image quality you are used to from your photo plate. MORADA is the first sidemounted camera whose features surpass film: the field of view is approximately twice that of film and it shows the same fine detail as film with the same field of view.

More details about the MORADA can be found on our web site:

\section{Morada}

11 MegaPixel side-mounted TEM camera

\section{www.soft-imaging.net/TEM}

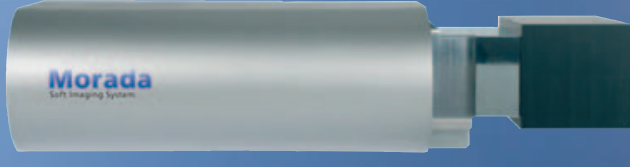

For detailed information please contact:

Soft Imaging System

info.de@soft-imaging.net

www.soft-imaging.net/tem

North America: (888) FIND SIS +1 (303) 234-9270

Europe: +49 (251) 79800-0

Asia | Pacific: +60 (3) 8313-1400 
The stability of the spectrum depends highly on the stability of the broadband Stokes light, which has a noise of below $5 \%$ within an hour. The dashed line in Figure 2a shows the nonresonant background, which accompanies the resonant CARS signal. In Figure $2 \mathrm{~b}$, the normalized CARS spectrum of benzonitrile is presented by taking the ratio between the resonant signal to the nonresonant background. Figure $2 \mathrm{c}$ is a spontaneous Raman spectrum of benzonitrile acquired in our instrument at a laser power of $23 \mathrm{~mW}$. To contrast the efficiencies of the two processes, the Raman spectrum required $1000 \mathrm{~ms}$ acquisition time to obtain a similar signal-to-noise ratio to the CARS spectrum taken in $17 \mathrm{~ms}$.
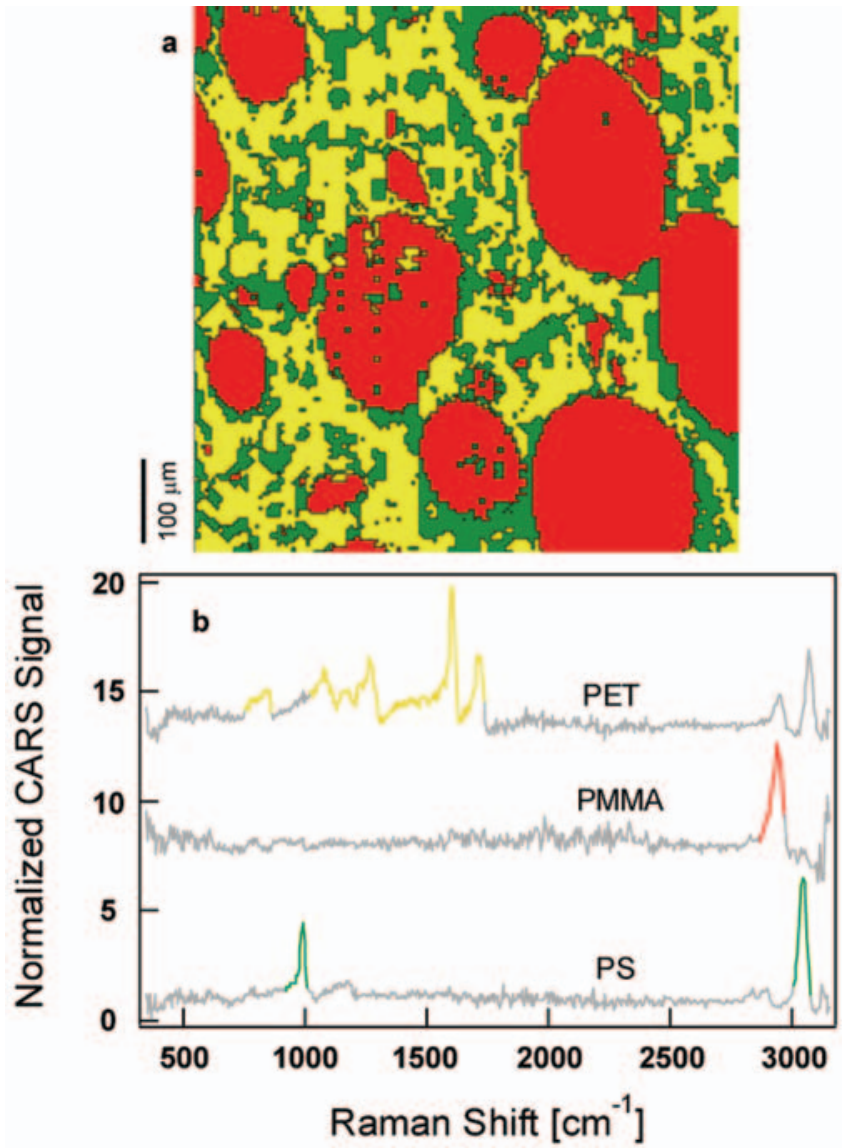

Figure 3. Panel a: Broadband CARS micrograph of a phase-separated polymer blend including equal parts of PMMA, PS, and PET. The colors red, green and yellow correspond to PMMA, PS, and PET respectively. Panel b: Reference spectra from each of the individual polymer components (with arbitrary vertical shift for clarity). The highlighted segments indicate spectral regions that were used for identification of spectra from each pixel in Panel a.

The ability of broadband CARS microscopy to perform chemically sensitive hyperspectral imaging is illustrated in Figure $3 \mathrm{a}$. The sample is a tertiary polymer blend containing equal parts of polystyrene (PS), poly(methyl methacrylate) (PMMA) and poly(ethylene terephthalate) (PET). The pseudo colors red, green and yellow in the image are assigned for PS, PET, and PMMA, respectively. The image contains $150 \times 150$ pixels and the dwell time at each pixel is 17 $\mathrm{ms}$. The CARS spectra for each of the components in the blend are given in Figure 3b. The highlighted regions in the spectra are used to assign the identity of a given pixel. By comparing the spectra, we can assign the polymer identity with a confidence level of $99 \%$.

We believe that broadband CARS microscopy has notable potential for developing into a widely used imaging technique. In order for this to happen, the following challenges must be overcome; some of these issues are specific to our instrument, while others are generic to broadband CARS microscopy. Frequency chirping of the Stokes light is a challenge that is specific to our instrument. Chirping occurs in our instrument due to the frequency dependent refractive index of the tapered fiber we use to generate broadband light. It results in temporal mismatch between the pump and some of the Stokes light, thereby decreasing the CARS signal in the corresponding spectral ranges. While frequency chirping is an issue in our instrument, there may be other approaches to broadband CARS in which frequency chirping is negligible. One possibility is to generate broadband Stokes light with a short photonic crystal fiber. The challenges that are generic to broadband CARS include increasing signal intensity by reducing axial chromatic aberration (ACA) of the focusing objective and minimizing the nonresonant background. A poor spatial overlap between the pump and Stokes light due to ACA of the microscope objective can significantly decrease the signal strength. Sensitivity to ACA is a strong function of numerical aperture. Fortunately, at NA $=0.8$, the requirement that ACA be less than $1 \mu \mathrm{m}$ over a spectral range of $800 \mathrm{~nm}$ to $1100 \mathrm{~nm}$ is not beyond the specifications of commercially available objectives. Thus, we were able to use a commercial $0.8 \mathrm{NA}$ objective in our first study.2 Of course, imaging at higher NA is desirable for increased signal and higher spatial resolution, and the ACA issue will have to be resolved. The presence of the nonresonant background is another major challenge in CARS microscopy because it imposes a lower boundary for detection limit. Reducing or eliminating the nonresonant background is therefore highly desirable. Efforts in addressing these issues are currently underway in our laboratory. We are reasonably confident that these issues will be resolved in the near future and an improved sensitivity and signal-to-noise ratio are within reach.

In the past few years, CARS microscopy has been enjoying increased attention and it is continuously generating strong interests in the field of biological imaging. Most recently, Xie et al. have used narrowband CARS microscopy to monitor lipid droplet trafficking in cell and differentiation of fibroblasts to adipocytes. ${ }^{4}$ The authors show that before fibroblasts fully differentiate into adipocytes by acquiring a large number of lipid droplets in cytoplasm, there exists a period in which almost all the cytoplasmic lipid droplets disappear. This result has never been observed before with other techniques. This work clearly indicates that the noninvasiveness, high sensitivity and selectivity of CARS present the promise of facilitating increased insights into many more biological processes. We are hopeful that the increase in chemical specificity through hyperspectral imaging with our microscope, in addition to the inherently superb spatial and temporal resolution of CARS, will allow the use of broadband CARS microscopy for noninvasively tracking the temporal and spatial course of cellular events involved in complex biological processes such as differentiation and signal transduction.

\section{Reference List}

1. Duncan, M. D.; Reintjes, J.; Manuccia, T. J. Opt. Lett. 1982, 7, 350.

2. Cheng, J. X.; Xie, X. S. J. Phys. Chem. B 2004, 108, 827.

3. Kee, T. W.; Cicerone, M. T. Opt. Lett. 2004, In press.

4. Nan, X. L.; Cheng, J. X.; Xie, X. S. J. Lipid Res. 2003, 44, 2202.

This manuscript is an official contribution of the National Institute of Standards and Technology. It is not subject to copyright in the United States. 

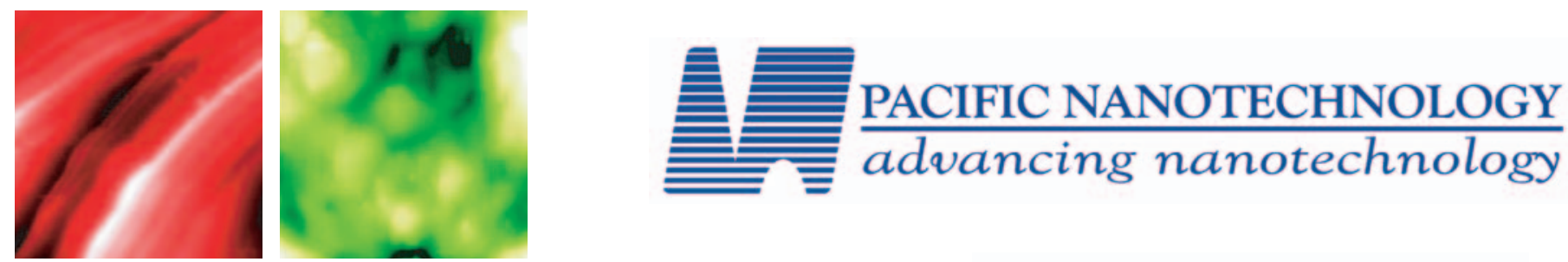

\section{Nano- R $^{\mathrm{TM}}$ Grystal Scanner ${ }^{\mathrm{TM}}$}
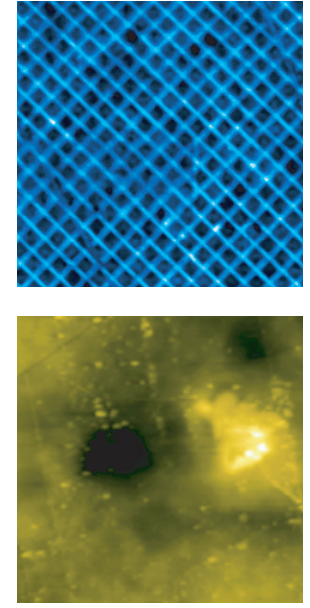

$1, \because \cdots 1,1, \cdots$ $\because \cdots 11,1,111$ $\because 11, \cdots \cdots$

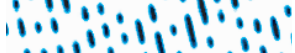
$11 \because \cdots 1,11,1,1$

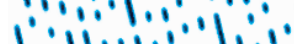
$1 \therefore 1, \ldots 1,1, . .1$
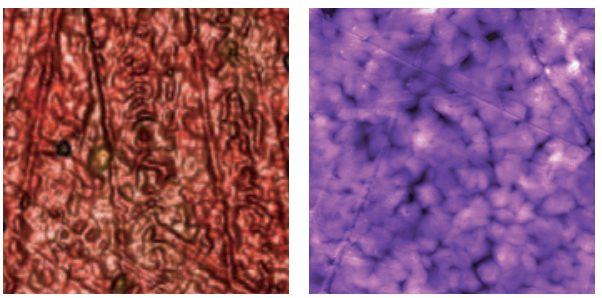

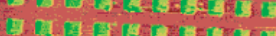

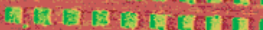

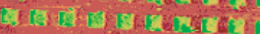

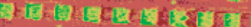

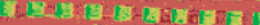

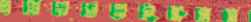
chy oryongen

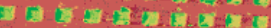

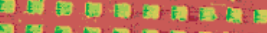
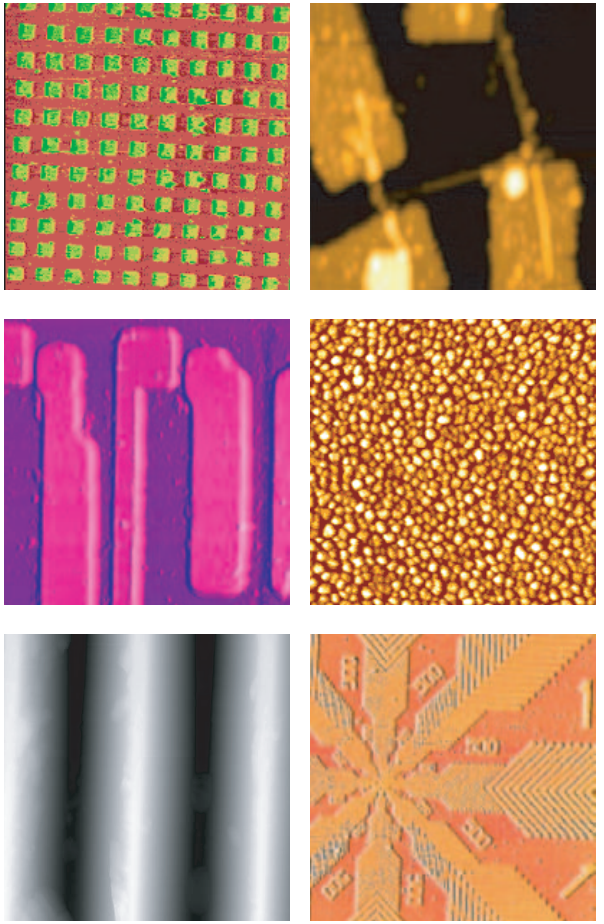

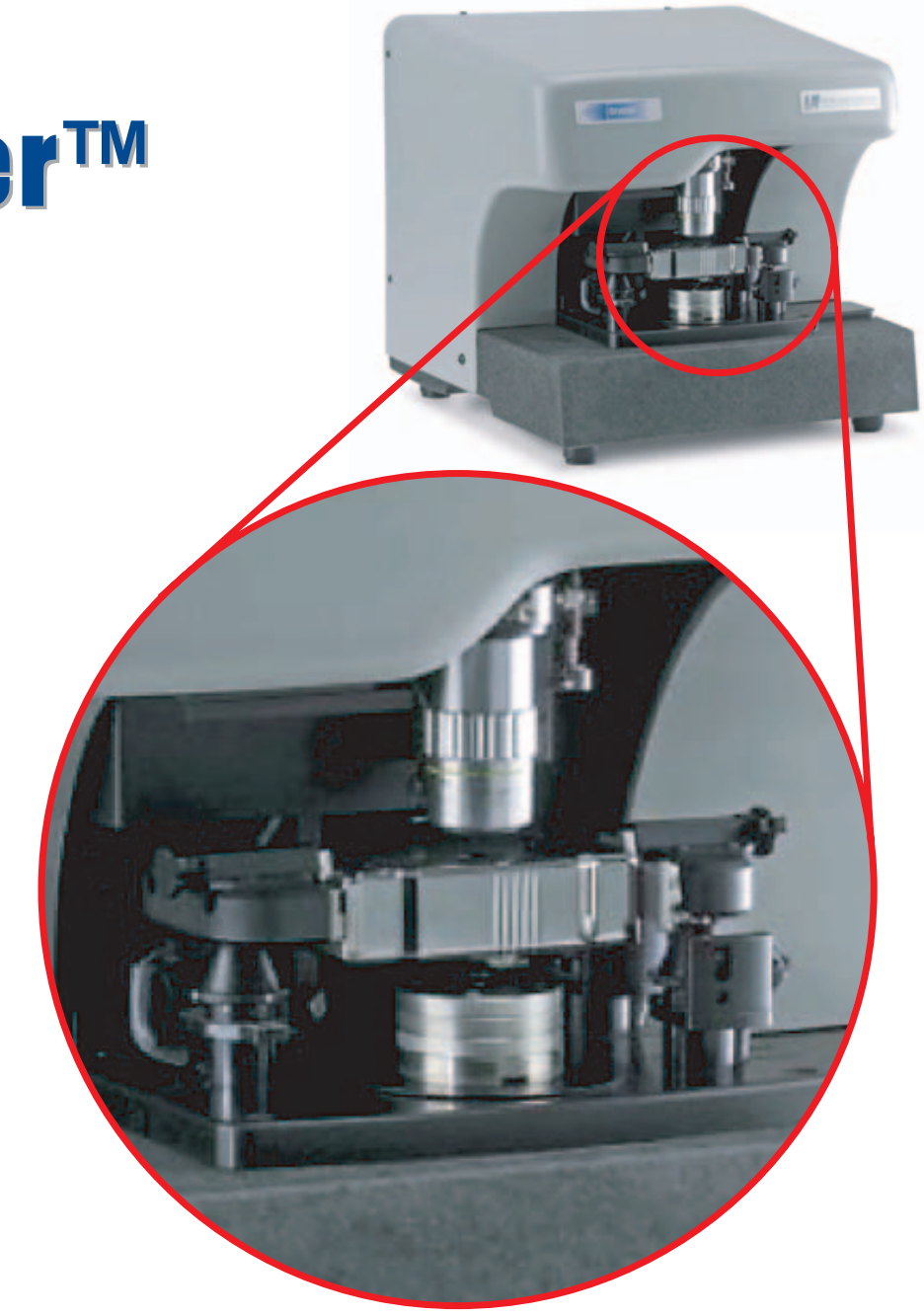

- Nano-R ${ }^{\mathrm{TM}}$ has a new scanner which makes topographic imaging simple

- Crystal Scanner uses Point \& Scan ${ }^{\mathrm{TM}}$ technology load the sample, the probe and just scan

- No complex operating procedures - no laser to adjust

- Motorized x,y stage and automated video microscope features make high resolution nanoscale imaging easy

(800) 246-3704

3350 Scott Blvd. \#29 - Santa Clara, CA 95054

Phone: (408) 982-9492 • Fax: (408) 982-9151

www.pacificnano.com • www.probestore.com 